\title{
Nanomedicine and its Applications
}

\section{Manu M*}

Department of Alumnus with Electrical Engineering, University of Bridgeport, Bridgeport, USA

*Corresponding author: Manu Mitra, Department of Alumnus with Electrical Engineering, University of Bridgeport, Bridgeport, United States, Email: mmitra@my.bridgeport.edu

\section{Editorial}

\section{Introduction}

Nanomedicine plays a vital role in drug delivery and regenerative medicines. Nanoparticles enable clinician to target drugs right at the root of disease, which not only increases efficiency but also minimizes side effects. They also offer new potential for controlled release of therapeutic substances [1].

\section{Editorial}

Volume 4 Issue 3

Received Date: November 19, 2019

Published Date: November 29, 2019

DOI: $10.23880 /$ nnoa-16000166

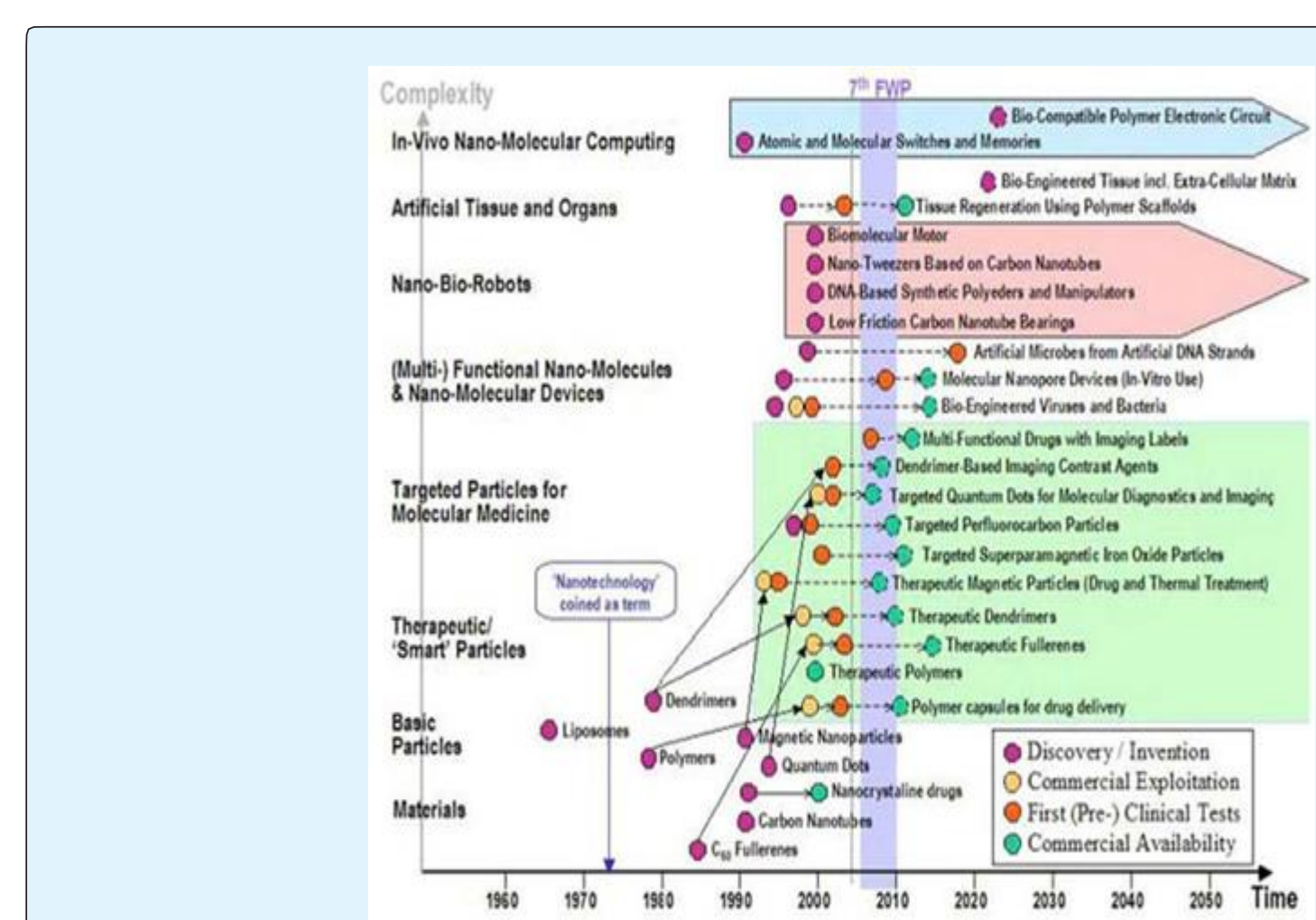

Figure 1: Illustrates indicate what has already been achieved and what the future prospects for nanomedicine [1] are. 


\section{Nanomedicine \& Nanotechnology Open Access}

\section{Nanomedicine for Anti-Cancer Chemotherapy}

Scientists at University of Helsinki in association with other university have developed a new technique for anticancer nanomedicine for cancer chemotherapy. This new nano tool supports novel approach to use cell based nanomedicines for efficient cancer chemotherapy.

Exosomes contain various molecular elements of the cell including proteins and RNA. Scientist utilized them together with synthetic nanomaterial as carrier's anticancer drugs [2,3].

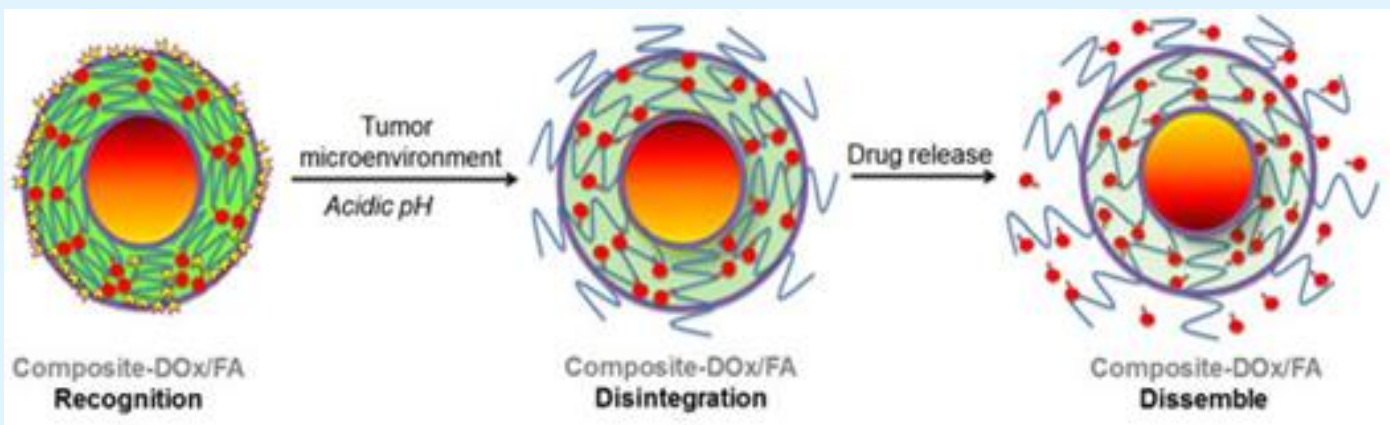

Figure 2: Illustrates the drug release mechanism via functional outcome of the $\mathrm{pH}$ response illustrated in the schematic diagram. Image Source: Smart Materials and Biodevice group, Linköping University [1].

\section{Nanomedicine Aims to Reach Areas like Brain}

As per researcher's study, they found a way to transport specific drugs to parts of the body that are very difficult to access with an ordinary drugs. In this study, Yshaped block catiomer (YBC) binds with definite therapeutic materials to constitute a package of size 18 nanometers wide. This bundle is less than one-fifth the size of those that were considered in previous studies; so it can pass through much smaller gaps. This allows nanomedicine to pass through barriers in cancers of the brain and pancreas $[4,5]$.

\section{Nanomedicine to Treat Brain Cancer}

For better treatment and advancing the technology one of the most serious form of brain cancer. Researchers from Illinois reported a novel method of first nanoparticle that can seek and destroy brain cancer cells without actually damaging neighboring healthy cells.

The solution contain chemically linked titanium dioxide nanoparticles to an antibody that identifies and attaches to GMB cells. In their experiment, these nanoparticles killed up to 80 percent of the brain cancer cells after 5 minutes of exposure to focused white light $[6,7]$.

Manu M. Nanomedicine and its Applications. Nanomed Nanotechnol 2019, 4(3): 000166.

\section{Nanomedicine Helps to Resolve Inflammation and Develops Tissue Healing}

A team of researchers at Columbia University Medical Center in collaboration with Massachusetts Institute of Technology and Brigham and Women's Hospital has created biodegradable nanoparticles that can deliver nano drug to the sites of tissue injury. It holds promise for the treatment of wide array of diseases characterized by excessive inflammation such as atherosclerosis [8].

\section{Machine Learning Technique Analyzes Nanomedicines for Cancer Therapy}

Scientists developed non-obvious and a novel technique to improve challenging nanoparticles bringing them one step closer for treatment option for many forms of cancer, genetic diseases, neurological disorders and other diseases.

In this research variation in structure leads to interdependent contributions to the efficacy. For instance, the ability to stimulate an immune response can depend on nanoparticle size and also illustrates how DNA molecules are oriented on the nanoparticle surface $[9,10]$. 


\section{Nanomedicine \& Nanotechnology Open Access}

\section{Conflicts of Interest}

There is no conflict of interest as per Author's point of view.

\section{References}

1. Nanowerk (2017) Nanotechnology in healthcare.

2. Yong T, Zhang $\mathrm{X}$, Bie $\mathrm{N}$, Zhang $\mathrm{H}$, Zhang $\mathrm{X}$, et al. (2019) Tumor exosome-based nanoparticles are efficient drug carriers for chemotherapy. Nature Communications 10(1): 3838.

3. University of Helsinki (2019) Novel anti-cancer nanomedicine for efficient chemotherapy.

4. Watanabe S, Hayashi K, Toh K, Kim HJ, Liu X, et al. (2019) In vivo rendezvous of small nucleic acid drugs with charge-matched block catiomers to target cancers. Nature Communications 10(1): 1894.

5. University of Tokyo (2019) New nanomedicine slips through the cracks, reaches brain: Nanomachines aim to deliver cancer drugs to hard-to-reach areas like the brain.
6. Rozhkova EA, Ulasov I, Lai B, Dimitrijevic NM, Lesniak MS, et al. (2009) A High-Performance Nanobio Photocatalyst for Targeted Brain Cancer Therapy. Nano Letters 9(9): 3337-3342.

7. American Chemical Society (2009) Toward a Nanomedicine for Brain Cancer.

8. Columbia University Medical Center (2013) New nanomedicine resolves inflammation, promotes tissue healing.

9. Yamankurt G, Berns EJ, Xue A, Lee A, Bagheri N, et al. (2019) Exploration of the nanomedicinedesign space with high-throughput screening and machine learning. Nature Biomedical Engineering 3(4): 318-327.

10. Northwestern University (2019) New machine learning technique rapidly analyzes nanomedicines for cancer immunotherapy: Novel approach will aid scientists in optimizing SNAs as therapeutic vaccines to treat cancer. 